University of Nebraska - Lincoln

DigitalCommons@University of Nebraska - Lincoln

Faculty Publications, Department of Psychology

Psychology, Department of

2004

\title{
Why be nice? Psychological constraints on the evolution of cooperation
}

Jeffrey R. Stevens

University of Nebraska-Lincoln, jstevens5@unl.edu

Marc D. Hauser

Harvard University

Follow this and additional works at: https://digitalcommons.unl.edu/psychfacpub

Part of the Psychiatry and Psychology Commons

Stevens, Jeffrey R. and Hauser, Marc D., "Why be nice? Psychological constraints on the evolution of cooperation" (2004). Faculty Publications, Department of Psychology. 542.

https://digitalcommons.unl.edu/psychfacpub/542

This Article is brought to you for free and open access by the Psychology, Department of at DigitalCommons@University of Nebraska - Lincoln. It has been accepted for inclusion in Faculty Publications, Department of Psychology by an authorized administrator of DigitalCommons@University of Nebraska - Lincoln. 


\title{
Why be nice? Psychological constraints on the evolution of cooperation
}

\author{
Jeffrey R. Stevens and Marc D. Hauser \\ Department of Psychology and Primate Cognitive Neuroscience Laboratory, Harvard University, Cambridge, MA 02I38, USA \\ Corresponding author - J. R. Stevens
}

\begin{abstract}
Animals often aid others without gaining any immediate benefits. Although these acts seem to reduce the donor's fitness, they are only apparently altruistic. Donors typically help because they or their kin receive future benefits or avoid costly punishment. Reciprocal altruism - alternating the roles of donor and recipient - has been a wellstudied form of cooperation among non-kin because of its intuitive appeal in explaining human cooperation. Despite immense theoretical interest, little empirical evidence substantiates the biological importance of reciprocal altruism in non-human animals. We propose that this is because psychological mechanisms constrain its application in cooperative contexts. In particular, we contend that cognitive limitations such as temporal discounting, numerical discrimination and memory make reciprocity difficult for animals.
\end{abstract}

Why be nice if you can benefit by being selfish? An$\checkmark$ swer: cooperation sometimes pays off. Numerous species cooperate in contexts such as foraging, mate attraction, predator avoidance, territory defense and parental care [1]. Among humans, in particular, cooperation seems to have been elevated to an integral part of society. Defining cooperation in economic terms - joint action for mutual benefit $[1,2]$-allows us to assess how costs and benefits, in terms of evolutionary fitness, influence the circumstances under which cooperation exists. For instance, in situations in which individuals gain immediate benefits by cooperating, cooperation is "selfish." Selfish cooperation, or mutualism [3], occurs when cooperators receive immediate, selfish benefits. Selfish cooperation is quite common in animal societies, particularly in cooperative hunting situations [4].

When cooperation is "altruistic" - costly to the cooperator and beneficial to the recipient-the temptation to cheat is high because defection (not cooperating) provides immediate benefits. So why help another at a cost to yourself? The simple answer is that what appears to be altruistic cooperation is only costly in the short term. Altruistic cooperators gain selfish benefits either by helping kin or by recouping their losses in future interactions. To accrue these selfish benefits, altruistic cooperators must use "conditional strategies" such as interacting only with relatives (kin selection) [5], interacting only with those that have cooperated previously (reciprocal altruism) [6], or receiving threats of sanctions (harassment or punishment) (see Box $1 ;[7,8])$.

In this article we focus on a form of altruistic cooperation with interesting psychological implications: reciprocal altruism (or reciprocity) - the alternation of donor and recipient roles in repeated altruistic interactions. When Trivers [6] introduced the concept of reciprocal altruism, he outlined necessary prerequisites, such as: (i) a large benefit to the recipient and a small cost to the donor; (ii) repeated opportunities for cooperative interaction; and (iii) the ability to detect cheaters. Instances of human cooperation satisfy these requirements and demonstrate the prevalence of reciprocal altruism across different economic contexts $[9,10]$ and cultures [11]. Some argue that reciprocity is so integral to human society that we have evolved specialized cognitive mechanisms to facilitate its stability, including the systematic detection and punishment of cheaters (see Box 1; $[12,13]$ ).

Given that reciprocity is common in humans and that the prerequisites appear trivial, should we expect to see it in non-human animals? We argue that the prerequisites for reciprocal altruism have been underestimated. A careful dissection reveals a host of underlying mechanisms necessary for both initiating a reciprocal relationship and for maintaining it over the long term. Some of the essential psychological ingredients for reciprocation include numerical quantification, time estimation, delayed gratification, detection and punishment of cheaters, analysis and recall of reputation, and inhibitory control; depending on the nature of the reciprocal interaction, some or all of these capacities may be necessary. If reciprocity is, indeed, as cognitively complex as we suggest, then we must anchor our theoretical predictions about adaptive function in realistic constraints imposed by neural and psychological design features. Because of these limitations, we predict that reciprocal altruism will be rare among animals, and when it appears, will represent a relatively minor force in the evolution of social organizations.

Here we provide a brief discussion of the evidence for reciprocal altruism in animals, make the claim that it is rare, and then offer some suggestions for why it is rare. Be- 


\section{Box I. Cheater detection and punishment}

\section{Cheater detection}

An important requirement of reciprocity is the ability to detect cheaters. Although cheating appears to be rampant in animals [4], detecting cheaters has not been well-studied. The case is different, however, for humans. Cosmides and Tooby [12] hypothesize that humans have specialized adaptations to assist in cheater detection. To test this they presented subjects with the Wason selection task-a simple test of logic in which subjects must determine whether the conditional rule "If $p$ then $q$ " has been violated. Cosmides and Tooby found that subjects often failed when presented with a version of this logic problem that used abstract propositions (e.g. matching particular letters with particular numbers). By contrast, when given a social-contract version of the test (e.g. enforcing the drinking age), the subjects performed much more accurately. Given these differences in accuracy, Cosmides and Tooby propose that humans have specialized cognitive modules devoted to detecting cheaters.

One significant piece of support for this "cheater detection module" hypothesis comes from a cognitive neuropsychological study [46]. In this study, a patient (R.M.) with severe bilateral damage to the orbitofrontal cortex, the temporal cortex and the amygdala (areas associated with social intelligence) was tested in a series of social-contract and "precaution" problems. In comparison with non-damaged subjects and subjects with severe damage in other areas, R.M. performed less well in social-contract scenarios, but they all performed equally well in the precaution problems. Cosmides and Tooby interpret these data as evidence for specialized brain areas associated with social reasoning.

cause the literature on reciprocity and its associated cognitive mechanisms is vast, our intent is not to review it, but to pinpoint a few reasons why reciprocal altruism is rare in non-human animals and common in humans.

\section{Reciprocal altruism: theoretical and empirical evidence}

Following Trivers, the concept of reciprocity remained all but untouched until Axelrod and Hamilton [14] presented a possible reciprocal strategy that allows for stable cooperation in the Prisoner's Dilemma scenario (see Box 2; [15]). Axelrod and Hamilton suggested that stable cooperation can emerge if the cooperative interactions occur repeatedly, the opening move is cooperative, and from that point on, each player copies the other's moves. This winning strategy is a version of reciprocity called Tit-For-Tat (TFT). Several theoretical investigations confirmed the efficacy of TFT, whereas others provided alternative strategies that could maintain cooperation (reviewed in [1]).

Several experimental and field studies of fish [16], vampire bats [17], primates [18-20] and other species have reported evidence of reciprocity. Although these studies elegantly show that there are naturally occurring contexts in which reciprocity might provide a solution to altruism among unrelated individuals, they are open to alternative explanations and several criticisms, including the fitness costs and benefits are ambiguous and the reciprocal events among non-kin are rare. Two laboratory experiments circumvent some of these criticisms by directly manipulating the costs and benefits of cooperation and controlling the partner's behavior.

Stephens and colleagues placed blue jays (Cyanocitta cristata) in experimental games such as the Prisoner's Dilemma in which pairs of birds peck keys to receive rewards $[2,21,22]$. Jays cooperated in these situations, but only in specialized circumstances, with no evidence of TFT-like strategies (Figure 1). Hauser and colleagues designed a se-

\section{Punishment}

Cheaters can be dealt with in two ways: retaliation and punishment. Retaliation is simply withholding future benefits from a cheater-that is, reciprocal defection, such as that found in TFT. Another way to manage cheating is to impose costs on defection by punishing cheaters. Clutton-Brock and Parker contend that punishment is a common technique used by animals to enforce cooperation [7]. Although little new evidence has emerged to support this contention, a related explanation-harassment-may explain some instances of enforced cooperation in animals $[8,47]$. In humans, however, there is a rich literature describing the use of punishment in cooperative situations [48-50]. Some economists and anthropologists suggest that humans might have evolved a different and apparently unique form of cooperation called "strong reciprocity" [50]. This combination of reciprocity and punishment has been defined by Gintis and colleagues as a "predisposition to cooperate with others and to punish those who violate the norms of cooperation, at personal cost, even when it is implausible to expect that these costs will be repaid either by others or at a later date" [49]. Proponents of this perspective argue that current models of human behavior that focus on self-interest cannot account for the frequency of human cooperation. They propose that human societies can be characterized by the special circumstances necessary to maintain cooperation via group selection. Regardless of whether punishment is self- or group-interested, it is nevertheless quite common in human cooperative situations and noticeably less common in animal societies.

ries of experiments in which cotton-top tamarins (Saguinus oedipus) could altruistically pull a tool to give food to an unrelated recipient without getting any food for themselves [23]. Tamarins pulled the tool most often for partners that always pulled and infrequently for partners that never pulled (Figure 2). The tamarins, however, cooperated less than $50 \%$ of the time, and as each game progressed, the amount of food given dropped. Like jays, tamarins can maintain some level of cooperation under some restricted conditions. Overall all, however, neither species demonstrates robust reciprocity.

\section{Box 2. Prisoner's dilemma}

In the Prisoner's Dilemma, two individuals simultaneously choose one of two strategies: cooperate or defect. The resulting payoff depends on both players' choices (Table I). To qualify as a Prisoner's Dilemma, the payoffs must conform to the following set of inequalities: $T>R>P>S$. Mutual cooperation results in a moderate reward $(R)$, but mutual defection leads to low payoffs for both players $(P)$. When one cooperates and the other defects, the defector receives the largest possible reward $(T)$ and the cooperator receives the smallest possible reward $(S)$. This implies that mutual cooperation is better than mutual defection, but for an individual player, there is a sizable temptation to defect. Therefore, the evolutionarily stable strategy in a one-shot game is defection.

Table I. Payoff matrix for Prisoner's Dilemma. The row player receives payoffs $R, T, P$, or $S$, depending on whether the column player cooperates $(C)$ or defects $(D)$. The player that defects when its opponent cooperates receives the maximum payoff of $T$ fitness units

\begin{tabular}{ll|ll} 
& & Against & \\
& & C & D \\
\hline Payoff to: & C & $R$ & $S$ \\
& D & $T$ & $P$
\end{tabular}




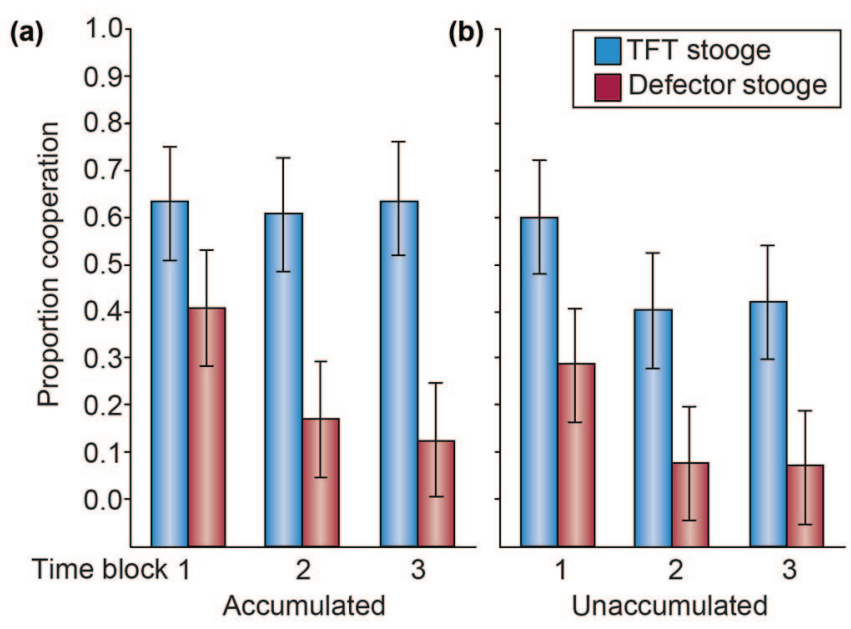

Figure I. Stephens et al. [22] tested blue jays in experimental Prisoner's Dilemma situations and recorded the proportion of trials in which they cooperated. Pairs of jays played repeated Prisoner's Dilemma games by landing on perches associated with cooperation or defection. The number of pellets released into each bird's food bin depended on the choices of both birds. In this particular experiment, food either accumulated over a series of trials in the Plexiglas tubes (a), or was delivered immediately following a trial (b). In addition, a freely behaving bird played against a stooge that either always defected (shown in red) or played Tit-For-Tat (TFT, shown in blue). The jays only cooperated consistently when food accumulated and they played against a TFT stooge, suggesting that discounting and opponent strategy influenced cooperation. (Adapted from [22], courtesy of American Association for the Advancement of Science).

\section{Why is reciprocity rare?}

Given the theoretical feasibility and ubiquity of human reciprocity, why do we find little evidence of non-human animals reciprocating [24]? Perhaps researchers have not used the appropriate combination of species and methodology to find reciprocity. We find this explanation unsatisfying because cooperation has been investigated in a large number of species, including invertebrates, fish, birds and many mammal species [1]. Researchers have also used several methodological techniques to investigate cooperation, ranging from observation to natural experiments to highly controlled laboratory experiments.

We propose that reciprocity is uncommon because it is too cognitively demanding for most, if not all, non-human animals. What started out as a simple solution to the problem of altruism among non-kin turns out to be much more challenging.

\section{Cognitive constraints on cooperation}

Cognitive abilities are clearly important in constraining animal behavior. To investigate how these constraints influence cooperation and reciprocity, we must break this problem down into its component parts. Here we examine a suite of cognitive abilities necessary to implement reciprocal strategies. There are likely to be constraints on time estimation, detection and punishment of cheaters, analysis and recall of reputation, and inhibitory control, but here we focus on temporal discounting, numerical discrimination, learning and memory because they are well studied in human and non-human animals and have intuitive links to cooperation.

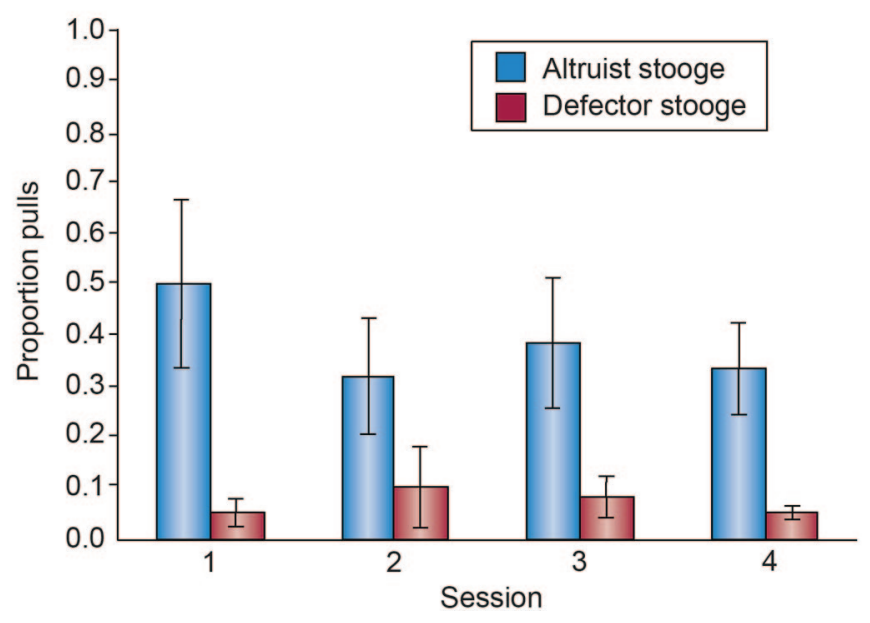

Figure 2. In Hauser et al. [23], pairs of tamarins alternated the roles of donor and recipient in this game of altruism. The donor had access to a tool that when pulled distributed food only to its partner. As in the jay example (Fig. I), freely behaving subjects played against stooges, but here they encountered either a unilateral altruist stooge (blue) or a unilateral defector stooge (red). Over all sessions, the tamarins pulled the tool for altruists much more frequently than for defectors, indicating that their cooperation was contingent on their partner's cooperation. (Reprinted with permission from [23]. Copyright 2003 Royal Society of London).

\section{Temporal discounting}

Temporal discounting is the devaluing of future rewards, which often results in a preference for smaller, immediate rewards over larger, delayed rewards. For example, imagine that a monkey encounters an unripe fruit-should it consume the fruit now or wait for it to ripen [25]? Waiting yields a larger benefit (more sugar available), but the future is uncertain-another monkey could eat it or a fungus might infest it, spoiling a perfectly good fruit. Uncertainties like these may have introduced a strong selective pressure to discount the future.

Given that the future is uncertain, should all organisms discount in the same way? Although discounting future rewards is probably universal among animals, the steepness of the discounting function (or "discounting rate") varies widely across species and contexts (Figure 3). A vast literature describes discounting in a broad range of species, using several different theoretical approaches, including delayed gratification [26] and rate maximization [25]. The discounting rate, however, has only been estimated for pigeons, rats, and humans [27-29]. Pigeons and rats both discount future rewards highly compared with humans (Figure 3).

Discounting is not necessarily a static parameter that applies to any choice situation. Rather, it can change choice preferences in different contexts. For example, in experimental situations, blue jays have a strong preference for immediate rewards [30]. In the wild during autumn, however, jays switch from consuming every acorn they encounter to caching them behind tree bark or under leaf litter. This example of context-specific discounting is common across several bird and mammal species and exemplifies the ability of animals to overcome constraints in specific ecological conditions. 


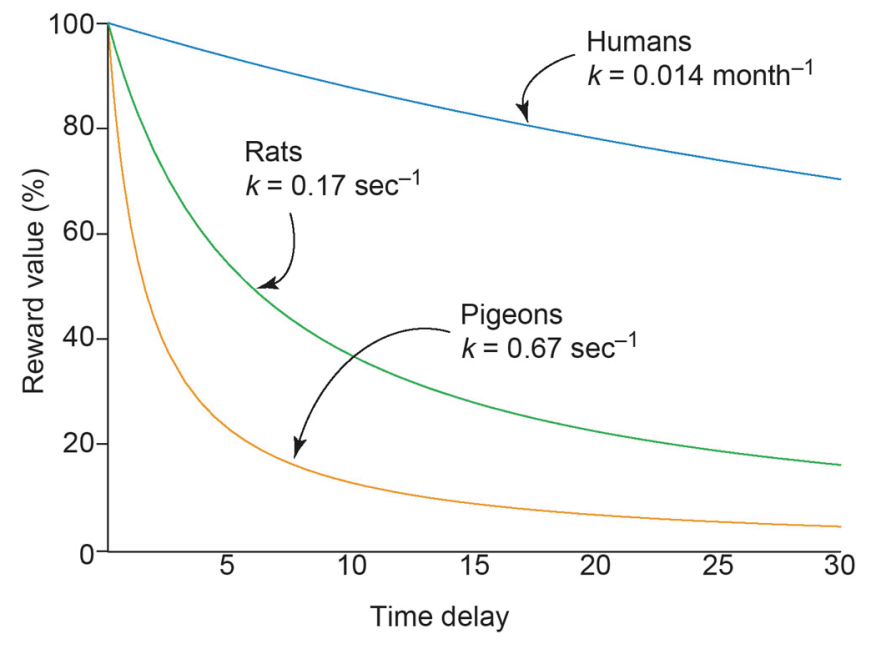

Figure 3. The discounting rate describes the steepness of the discounting function - that is, how quickly the reward is devalued over time. The hyperbolic model of discounting is described by $V=A /(I+k D)$, where $V$ is the subjective value of the reward, $A$ is the amount of the reward, $D$ is the delay to reward, and $k$ is a free parameter describing the discounting rate. This discounting rate $k$ has been estimated for pigeons and rats, suggesting that both species rapidly devalue food delayed in a matter of seconds $[27,28]$. Similar experiments on humans suggest that we devalue money at a much lower rate, on the order of months rather than seconds [29]. (Note that the Time delay axis has dual units. Discounting functions plotted from $k$ values reported in Mazur [27], Richards et al. [28], and Rachlin et al. [29]).

Many psychologists consider the iterated Prisoner's Dilemma to be an extension of the discounting problem [31, 32]. Individuals can choose between the immediate reward of defecting or the long-term reward of cooperating. Experimental data on variation in human discounting and cooperation validate this view. Discounting correlates with cooperation such that individuals who highly devalue future rewards cooperate less frequently [33]. In parallel, blue jays show stable cooperation in the Prisoner's Dilemma when the discounting rate is lowered by allowing payoffs to accumulate over several trials (Figure 1; [22]). In summary, because reciprocity requires paying an immediate cost for future benefits, the expected future benefit must be timediscounted appropriately for reciprocity to work.

\section{Numerical discrimination}

A challenge for reciprocal interactions lies in quantifying the economics of the entities given and returned and evaluating whether the exchange was equitable. For example, if an altruist gives four apples and receives one back, this is not equitable, and natural selection should eliminate this poor decision-maker from the population. Do animals count or quantify in these ways? If they do not, then either individuals are satisfied with some return, regardless of amount, or they are open to defectors giving back less than a fair amount.

Rats and pigeons can be trained to press a key a certain number of times for food. However, animals make more errors as the required number of presses increases (reviewed in [34]). When an experimenter requires a rat to press a key four times for food, it is usually exactly right, pressing four most of the time and on occasion pressing three or five times. By contrast, when the target number is 24 , the rat sometimes presses 24 times, but often it presses somewhere between 20 and 30 times. These studies show that animals can quantify number, but only approximately so.

Studies of foraging in animals show that individuals attempt to maximize the rate of energetic returns, choosing patches with more food over those with less [35]. As estimates of rates of return depend on quantifying amount of food consumed over time, we can ask whether animals count the pieces, estimate the volume, or time the foraging periods in a patch. When given a choice between two different numbers of food items, rhesus monkeys routinely choose the larger when both items are less than four, even when time and volume are controlled. Above four, however, the monkeys have difficulty discriminating unless the difference between the two numbers is large [36].

Based on an overwhelming number of carefully controlled experiments, it is now fair to say that animals have a number sense consisting of two naturally available systems [37]. One allows animals to count up to about four with precision; the second allows them to approximate large numbers. If animals engage in a bout of reciprocal altruism, they will either be limited to small numbers of objects in cases where the exchange must be precise (a banana for a banana), or they will be freed from this constraint where approximate exchanges are tolerated. Thus, when animals reciprocally exchange precise amounts of a resource, they must be able to quantify those amounts or they will be susceptible to cheaters.

\section{Learning/memory}

One of the primary alternative strategies to TFT is a learning-based strategy called win-stay/lose-shift or Pavlov [38]. This strategy is loosely based on Thorndike's law of effect [39], in that when an individual receives rewarding payoffs ( $T$ or $R$ ), it will repeat that choice, but if it receives punishing payoffs $(P$ or $S)$, it will switch choices (see Box 2). Nowak and Sigmund conducted evolutionary simulations of an iterated Prisoner's Dilemma and showed that Pavlov could emerge and remain stable in a stochastic environment [38]. The stability of Pavlov, however, depends on the actual values of $T, R, P$, and $S$. The threshold between rewards and punishments is critical in analyzing cooperative games [40]. If the threshold separates $T$ and $R$ from $P$ and $S$, the Pavlov strategy can work well.

In some of the earliest experimental work on the Prisoner's Dilemma using human subjects, Rapaport and Chammah [15] used negative payoffs for $P$ and $S$ and found relatively high levels of cooperation. By contrast, subjects cooperate much less in experiments using non-negative payoffs [41]. Zero payoffs also have potential effects on cooperation. Stephens and Clements [40] tested blue jays in payoff matrices with $S=0$ and $S=1$. In both cases the jays dropped to low levels of cooperation; however, when $S=0$ the jays dropped faster and to lower levels. Therefore, the relationship between thresholds and payoffs influenced learning rates, which, in turn affected cooperative behavior.

Limitations in memory decay, interference and capacity also constrain the frequency of reciprocity. Models of 
forgetting predict exponential or power functions [42], because memories decay rapidly over time. Therefore, longer time intervals between cooperative acts should make reciprocity more difficult. Time is not the only factor that potentially influences how memory interacts with cooperation. The presence of other types of computationally intensive events also interferes with memory. Milinksi and Wedekind tested working memory constraints on humans playing iterated Prisoner's Dilemma games [43]. Subjects played either a series of Prisoner's Dilemma games or a series of Prisoner's Dilemma games separated by a game of Memory. Although the overall outcomes did not differ between the two treatments, the strategies used by the subjects did differ: subjects without interference used memory-intensive Pavlov-like strategies, whereas subjects with interference used a generous version of TFT.

Related to the effect of interference on memory are capacity constraints. Even if the time between cooperative interactions is short and few distractions exist, every potential new partner increases the computational load of tracking the debts owed and favors given. Keeping score of reciprocal obligations with multiple individuals can be a computationally intensive burden on memory. Although few studies examine learning and memory constraints in animal cooperation, human studies suggest that these constraints can pose challenges for maintaining stable cooperative relationships. In summary, when the delay to reciprocation is long, both the donor and receiver are likely to forget the debt.

\section{Conclusions and prospects}

Cooperation is quite common in both human and nonhuman animal societies [1]. We argue, however, that most instances of animal cooperation can be attributed to either selfish or indirect benefits via mutualism and helping kin. We suggest that reciprocal altruism among unrelated individuals is rare if not absent among animals, despite its ubiquity in humans. In cases where it occurs in the laboratory, it is unclear whether the patterns observed would generalize to more natural and less controlled situations. We propose that cognitive constraints on temporal discounting, numerical discrimination, learning and memory, and other components, limit the ability of many species to implement and maintain reciprocally altruistic strategies. If this is correct, then comparative research should illuminate which components are shared with other animals, which are unique to humans, and why certain components evolved in our species and no other.

In addition, this framework can guide research into the psychological capacities mediating cooperation. Specifically, we propose that investigations of reciprocity must first evaluate the limitations of animals in the cognitive areas that we have described. What are the numerical discrimination abilities, discounting rates, learning rates, and memory features of the species being investigated? When testing reciprocity, researchers should consider these constraints in designing appropriate experiments. The next step is to compare these circumstances with naturally cooperative situations. Do natural cooperative contexts fall within the range of the species' cognitive abilities or are the constraints too restrictive to maintain reciprocity in the wild?
Another path into these cognitive problems is to look into the brain to search for neural correlates of cooperative behavior. Because there are no explicit studies of the neurobiology of cooperation in animals, we rely on neuroeconomics - the neurobiology of economic decision making in humans - with the hope that it will shed some light on the possible neural correlates of social interactions in animals $[44,45]$. As neuroeconomics is in its infancy, there is as yet no clear computational theory predicting how cooperation is processed and represented in the brain (but see [9]). However, if we can document the necessary and sufficient circuitry underlying human cooperation and reciprocity, then this will illuminate one way in which one species evolved this form of cooperation. Although other animals might solve this problem by means of other circuitry, if it turns out that animals are incapable of maintaining reciprocally stable relationships, then understanding which part of the circuitry is missing or deficient may help explain why.

The study of altruistic cooperation is clearly a cross-disciplinary endeavor, integrating behavioral ecology, evolutionary biology, experimental economics, neurobiology and psychology. Ultimately, understanding the nature of cooperation will require cooperation among these and other fields.

Acknowledgments-This work was supported by an NIHNRSA fellowship to J.R.S. and an NSF-ROLE grant to M.D.H. We thank Fiery Cushman, Alex Kacelnik, Robert Seyfarth, and the anonymous reviewers for comments on the manuscript.

\section{References}

1. L. A. Dugatkin. Cooperation Among Animals: An Evolutionary Perspective, Oxford University Press (1997).

2. K. C. Clements and D. W. Stephens, Testing models of nonkin cooperation: mutualism and the prisoner's dilemma. Anim. Behav. 50 (1995), pp. 527-535.

3. J. L. Brown, Cooperation-a biologist's dilemma. In: J. S. Rosenblatt, Editor, Advances in the Study of Behaviour, Academic Press (1983), pp. 1-37.

4. C. Packer and L. Ruttan, The evolution of cooperative hunting. Am. Nat. 132 (1988), pp. 159-198.

5. W. D. Hamilton, The genetical evolution of social behaviour (I, II). J. Theor. Biol. 7 (1964), pp. 1-52.

6. R. L. Trivers, The evolution of reciprocal altruism. Q. Rev. Biol. 46 (1971), pp. 35-57.

7. T. H. Clutton-Brock and G. A. Parker, Punishment in animal societies. Nature 373 (1995), pp. 209-216.

8. J. R. Stevens and D. W. Stephens, Food sharing: a model of manipulation by harassment. Behav. Ecol. 13 (2002), pp. 393-400.

9. K. A. McCabe, A cognitive theory of reciprocal exchange. In: E. Ostrom and J. Walker, Editors, Trust and Reciprocity: Interdisciplinary Lessons from Experimental Research, Russell Sage Foundation (2003), pp. 147-169.

10. E. Fehr and U. Fischbacher, The nature of human altruism. Nature 425 (2003), pp. 785-791.

11. J. Henrich et al., In search of Homo economicus: behavioral experiments in 15 small-scale societies. Am. Econ. Rev. 91 (2001), pp. 73-78. 
12. L. Cosmides and J. Tooby, Cognitive adaptations for social exchange. In: J. H. Barkow et al. The Adapted Mind: Evolutionary Psychology and the Generation of Culture, Oxford University Press (1992), pp. 163-228.

13. E. Fehr and S. Gächter, Altruistic punishment in humans. Nature 415 (2002), pp. 137-140.

14. R. Axelrod and W. D. Hamilton, The evolution of cooperation. Science 211 (1981), pp. 1390-1396.

15. A. Rapoport and A. N. Chammah. Prisoner's Dilemma: A Study in Conflict and Cooperation, University of Michigan Press (1965).

16. M. Milinski, Tit for Tat in sticklebacks and the evolution of cooperation. Nature 325 (1987), pp. 433-435.

17. G. S. Wilkinson, Reciprocal food sharing in the vampire bat. Nature 308 (1984), pp. 181-184.

18. F. B. M. de Waal, Food sharing and reciprocal obligations among chimpanzees. J. Hum. Evol. 18 (1989), pp. 433-459.

19. C. Packer, Reciprocal altruism in Papio anubis. Nature 265 (1977), pp. 441-443.

20. R. M. Seyfarth and D. L. Cheney, Grooming, alliances and reciprocal altruism in vervet monkeys. Nature 308 (1984), pp. 541-543.

21. Stevens, J. R. and Stephens, D. W. The economic basis of cooperation: trade-offs between selfishness and generosity. Behav. Ecol. (in press).

22. D. W. Stephens et al., Discounting and reciprocity in an Iterated Prisoner's Dilemma. Science 298 (2002), pp. 2216-2218.

23. M. D. Hauser et al., Give unto others: genetically unrelated cotton-top tamarin monkeys preferentially give food to those who altruistically give food back. Proc. R. Soc. Lond. B. Biol. Sci. 270 (2003), pp. 2363-2370.

24. P. Hammerstein, Why is reciprocity so rare in social animals? A protestant appeal. In: P. Hammerstein, Editor, Genetic and Cultural Evolution of Cooperation, MIT Press (2003), pp. 83-94.

25. A. Kacelnik, The evolution of patience. In: G. Loewenstein et al. Time and Decision: Economics and Psychological Perspectives on Intertemporal Choice, Russell Sage Foundation (2003), pp. 115-138.

26. W. Mischel et al., Delay of gratification in children. Science 244 (1989), pp. 933-938.

27. J. E. Mazur, An adjusting procedure for studying delayed reinforcement. In: M. L. Commons et al. Quantitative Analyses of Behavior: The Effect of Delay and of Intervening Events on Reinforcement Value Vol. 5, Lawrence Erlbaum (1987), pp. 55-73.

28. J. B. Richards et al., Determination of discount functions in rats with an adjusting-amount procedure. J. Exp. Anal. Behav. 67 (1997), pp. 353-366.

29. H. Rachlin et al., Subjective probability and delay. J. Exp. Anal. Behav. 55 (1991), pp. 233-244.

30. D. W. Stephens and C. M. McLinn, Choice and context: testing a simple short-term choice rule. Anim. Behav. 66 (2003), pp. 59-70.
31. L. Green et al., Prisoner's dilemma and the pigeon: control by immediate consequences. J. Exp. Anal. Behav. 64 (1995), pp. 1-17.

32. H. Rachlin. The Science of Self-Control, Harvard University Press (2000).

33. A. C. Harris and G. J. Madden, Delay discounting and performance on the prisoner's dilemma game. Psychol. Rec. 52 (2002), pp. 429-440.

34. S. T. Boysen and E. J. Capaldi. The Development of Numerical Competence: Animal and Human Models, Lawrence Erlbaum (1993).

35. D. W. Stephens and J. R. Krebs. Foraging Theory, Princeton University Press (1986).

36. Hauser, M. D. and Spelke, E. S. (in press) Evolutionary and developmental foundations of human knowledge: a case study of mathematics. In The Cognitive Neurosciences III (Gazzaniga, M., ed. ), MIT Press.

37. S. Dehaene. The Number Sense, Oxford University Press (1997).

38. M. A. Nowak and K. Sigmund, A strategy of win-stay, lose-shift that outperforms tit-for-tat in the Prisoner's Dilemma game. Nature 364 (1993), pp. 56-58.

39. E. L. Thorndike. Animal Intelligence, Macmillan (1911).

40. D. W. Stephens and K. C. Clements, Game theory and learning. In: L. A. Dugatkin and H. K. Reeve, Editors, Game Theory and Animal Behavior, Oxford University Press (1998), pp. 239-260.

41. A. M. Colman. Game Theory and its Applications in the Social and Biological Sciences, Routledge (1999).

42. S. Sikstrom, Forgetting curves: implications for connectionist models. Cogn. Psychol. 45 (2002), pp. 95-152.

43. M. Milinski and C. Wedekind, Working memory constrains human cooperation in the Prisoner's Dilemma. Proc. Natl. Acad. Sci. U.S.A. 95 (1998), pp. 13755-13758.

44. Stevens, J. R., and Hauser, M. D. (in press) Cooperative brains: psychological constraints on the evolution of altruism. In From Monkey Brain to Human Brain (Dehaene, S. et al., eds), MIT Press.

45. C. F. Camerer, Behavioural studies of strategic thinking in games. Trends Cogn. Sci. 7 (2003), pp. 225-231.

46. V. E. Stone et al., Selective impairment of reasoning about social exchange in a patient with bilateral limbic system damage. Proc. Natl. Acad. Sci. U.S.A. 99 (2002), pp. 11531-11536.

47. Stevens, J. R. and Gilby, I. C. A conceptual framework for non-kin food sharing: timing and currency of benefits. Anim. Behav. (in press).

48. E. Fehr and S. Gächter, Cooperation and punishment in public goods experiments. Am. Econ. Rev. 90 (2000), pp. 980-994.

49. H. Gintis et al., Explaining altruistic behavior in humans. Evol. Hum. Behav. 24 (2003), pp. 153-172.

50. R. Boyd et al., The evolution of altruistic punishment. Proc. Natl. Acad. Sci. U.S.A. 100 (2003), pp. 3531-3535. 